\title{
Restrictions on the four vector boson vertex in a weakly interacting standard model
}

\author{
Leo P.H.M. de Wit \\ Randall Laboratory of Physics, University of Michigan, Ann Arbor, MI 48109, USA
}

Received 19 July 1990

\begin{abstract}
There is no experimental evidence that the four vector boson ( $4 \mathrm{~W})$ vertex predicted by the standard model exists. To study the effects of this vertex, we introduce the parameter $\kappa$ such that $\kappa^{2}$ is the new $4 \mathrm{~W}$ coupling constant. We set constraints on $\kappa$ by considering unitarity requirements for vector boson scattering amplitudes. We find that the $4 \mathrm{~W}$ vertex must exist if the vector bosons interact weakly up to $\sqrt{s} \approx 300-350 \mathrm{GeV}$.
\end{abstract}

\section{Introduction}

At the time, the $S U(3) \times S U(2) \times U(1)$ standard model is widely accepted as the best theory to describe elementary processes. However, the signatures of some parts of the theory have not been observed yet (for example the top quark, the vector boson self-interactions and the Higgs boson).

In this paper, we will focus on the vector boson interactions. The standard model predicts that vector bosons can interact via 4-point vertices, but there is no evidence that such vertices are needed to build a unitary low energy theory. In this context, we determine at what energies vector boson 4-point vertices have to be introduced in such a theory.

The techniques used are essentially the same as in ref. [1]. We consider the tree amplitudes for vector-bosonvector-boson scattering. The energy at which the $J=0$ partial waves formed from these amplitudes saturate unitarity, sets a constraint on the form of the $4 \mathrm{~W}$ vertices in the theory. It is found that $4 \mathrm{~W}$ vertices are necessary if the theory is to interact weakly up to $\sqrt{s} \approx 300-350 \mathrm{GeV}$.

The outline of the paper is as follows. In section 2 , we define our model. In section 3 , we show how to put constraints on the 4-point vertex, and do a simple evaluation in the limit $m^{2} \gg s \gg M^{2}$. Section 4 describes how the exact calculation for all energy ranges is done. Section 5 gives the results of this calculation and section 6 is a discussion of these results.

\section{The model}

We want to determine what the contribution of the $4 \mathrm{~W}$ vertex to the vector-boson-vector-boson scattering amplitude is. To achieve this, we build the following model. We take the standard model lagrangian [2] and multiply the terms containing four vector bosons (but no photons) by a factor $\kappa$, keeping all other terms the same. From this lagrangian, we then form the Feynman rules in the usual way.

Obviously, taking $\kappa$ different from 1 breaks the SU(2) [not the U(1)] gauge invariance. Therefore, we no longer have the freedom to choose the gauge. The theory must be unitary, so we have to work in the unitary gauge. The theory is still non-renormalizable, but this does not affect our tree level analysis. The relevant parameters of the theory are $g$, the weak coupling constant; $s_{\theta}, c_{\theta}$, the sine and cosine of the weak mixing angle; $M$, the 
mass of the charged vector bosons $\mathrm{W}^{+}, \mathrm{W}^{-} ; m$, the mass of the Higgs boson $\mathrm{H}$. The mass of the neutral vector boson $\mathrm{W}^{0}$ is $M / c_{\theta}$ (at tree level). The values used for these parameters are $g^{2}=0.40, s_{\theta}^{2}=0.23, M=81 \mathrm{GeV}$.

\section{Constraints on the $J=0$ partial waves}

Consider the tree amplitudes $T$ for the various vector-boson-vector-boson scattering processes. These amplitudes can be decomposed in partial waves:

$T(s, t)=16 \pi \sum_{j=0}^{\infty}(2 j+1) a_{j}(s) P_{j}(\cos \theta)$,

where $s$ and $t$ are the usual Mandelstam variables, $\theta$ is the scattering angle. It can be shown from unitarity considerations that [3]

$\left|\Re\left\{a_{j}(s)\right\}\right| \leqslant \frac{1}{2}$.

These constraints set limits on $\kappa$ for a given energy. We find that the longitudinal $J=0$ partial wave amplitude sets the tightest constraint. Therefore, we only use

$\left|\Re\left\{a_{0}^{\mathbf{L}}(s)\right\}\right| \leqslant \frac{1}{2}$.

There are three types of tree diagrams, as shown in fig. 1. (Crossed diagrams must be included.) In general, their contributions to the $J=0$ partial wave amplitude are

(1) 4-point vertex diagram

$A s^{2}+B s$,

(2) vector boson exchange diagram

$C s^{2}+D s+E$,

(3) Higgs exchange diagram

$F s+G$.

Here $A, B, C, D$, and $F$ are constants, not containing kinematical variables. $E$ and $G$ play a significant role only when the Higgs is extremely heavy $(m \gg 2 \mathrm{TeV})[1,4]$, or when the energy $\sqrt{s}$ is close to the Higgs mass.

Apart from these effects, i.e. for $m^{2} \gg s \gg M^{2}$, the $s^{2}$ and $s$ terms are absent in the partial wave if

$A+C=0, B+D+F=0$.

This, however, is satisfied only if $\kappa$ is exactly 1 . If $\kappa$ is different from 1 , then unitarity is violated above a certain energy, regardless of the Higgs mass.

Away from the Higgs pole, the leading terms in the $J=0$ partial wave scattering amplitude for $\mathrm{W}_{\mathrm{L}}^{+} \mathrm{W}_{\mathrm{L}}^{-} \rightarrow$ $\mathrm{W}_{\mathrm{L}}^{+} \mathrm{W}_{\mathrm{L}}^{-}$are quadratic in $s$. These terms come from the diagrams given in fig. 2.

Combined, they result in the partial wave amplitude
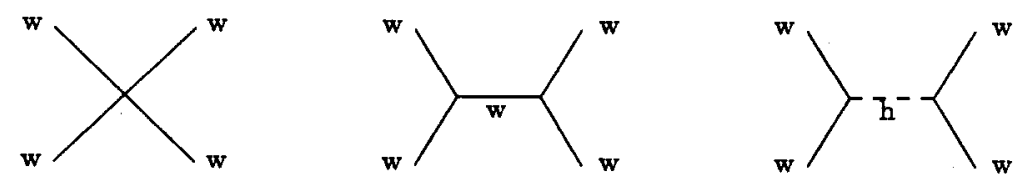

Fig. 1. Different types of diagrams contributing to WW scattering. 

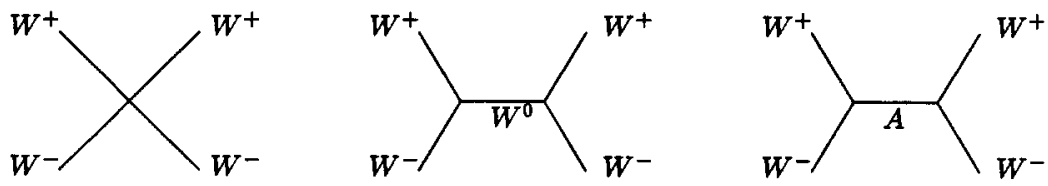

Fig. 2. Leading diagrams for $\mathrm{W}^{+} \mathrm{W}^{-} \rightarrow \mathrm{W}^{+} \mathrm{W}^{-}$scattering.

$a_{0}^{\mathrm{L}}(s)=\frac{g^{2} s^{2}}{96 \pi M^{4}}(1-\kappa)$.

It follows from eq. (3) that

$|1-\kappa| \leqslant \frac{48 \pi M^{4}}{g^{2} s^{2}}$

From this, bounds on $\kappa$ can be extracted. The higher the energy $\sqrt{s_{\text {weak }}}$ at which the theory is weak and unitarity is not violated, the closer $\kappa$ is to one. In fig. 3, the allowed values for $\kappa$ are plotted using eq. (9). The highest energy at which the $4 \mathrm{~W}$ vertex is not necessary to maintain unitarity, i.e. $\kappa=0$, is denoted by $\sqrt{s_{\text {crit }} \text { : }}$ :

$\sqrt{s_{\text {crit }}} \equiv\left(\frac{48 \pi}{g^{2}}\right)^{1 / 4} M \simeq 360 \mathrm{GeV}$.

It follows, that if $\sqrt{s}>\sqrt{s_{\text {crit }}}, \kappa$ is bounded by positive numbers, and a $4 \mathrm{~W}$ vertex is necessary.

These bounds can be refined by coupling the channels $W_{L}^{+} W_{L}^{-}$and $W_{L}^{0} W_{L}^{0}$ [1]. This is done by considering the following $2 \times 2$ matrix:

$\mathscr{M}=\left(\begin{array}{cc}a_{0}^{\mathrm{L}}\left(\mathrm{W}_{\mathrm{L}}^{+} \mathrm{W}_{\mathrm{L}}^{-} \rightarrow \mathrm{W}_{\mathrm{L}}^{+} \mathrm{W}_{\mathrm{L}}^{-}\right) & (1 / \sqrt{2}) a_{0}^{\mathrm{L}}\left(\mathrm{W}_{\mathrm{L}}^{+} \mathrm{W}_{\mathrm{L}}^{-} \rightarrow \mathrm{W}_{\mathrm{L}}^{0} \mathrm{~W}_{\mathrm{L}}^{0}\right) \\ (1 / \sqrt{2}) a_{0}^{\mathrm{L}}\left(\mathrm{W}_{\mathrm{L}}^{0} \mathrm{~W}_{\mathrm{L}}^{0} \rightarrow \mathrm{W}_{\mathrm{L}}^{+} \mathrm{W}_{\mathrm{L}}^{-}\right) & \frac{1}{2} a_{0}^{\mathrm{L}}\left(\mathrm{W}_{\mathrm{L}}^{0} \mathrm{~W}_{\mathrm{L}}^{0} \rightarrow \mathrm{W}_{\mathrm{L}}^{0} \mathrm{~W}_{\mathrm{L}}^{0}\right)\end{array}\right)$.

The unitarity condition becomes

$\mid \mathfrak{R}\{$ largest eigenvalue of $\mathscr{M}\} \mid \leqslant \frac{1}{2}$.

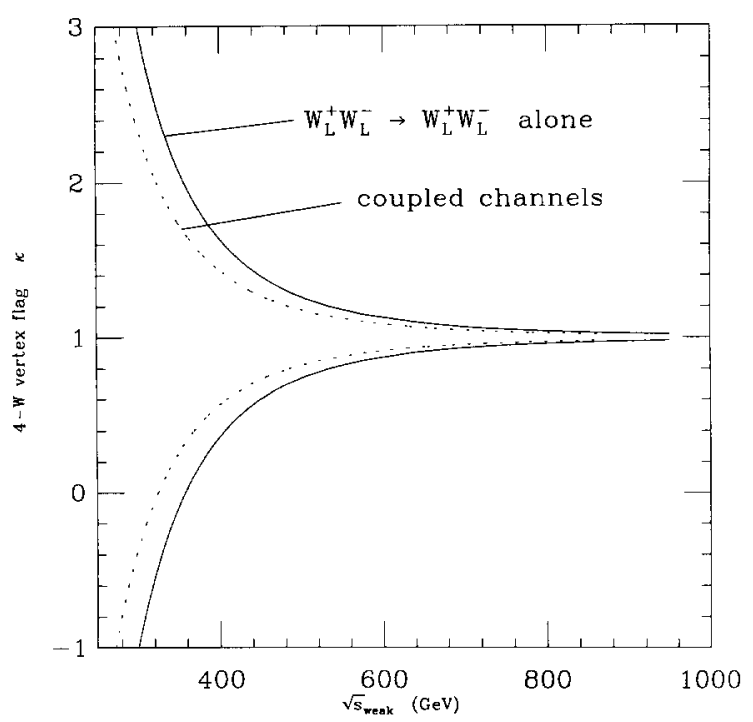

Fig. 3. Limits on $\kappa$ from the high energy calculation. 
In the case at hand, the matrix is

$\mathscr{M}=\frac{g^{2} s^{2}(1-\kappa)}{96 \pi M^{4}}\left(\begin{array}{cc}1 & \sqrt{2} c_{\theta}^{4} \\ \sqrt{2} c_{\theta}^{4} & 0\end{array}\right)$.

So, (12) becomes

$\frac{g^{2} s^{2}}{96 \pi M^{4}} \frac{1+\sqrt{1+8 c_{\theta}^{8}}}{2}|1-\kappa| \leqslant \frac{1}{2}$.

This relation is represented by the dotted lines in fig. 3 . The critical energy is

$\sqrt{s_{\text {crit }}} \simeq 320 \mathrm{GeV}$.

\section{The exact calculation}

We want to obtain more precise limits on $\kappa$ by calculating the exact $J=0$ partial waves from our model.

There is a fundamental problem in calculating exact partial wave amplitudes for WW scattering. For a process with a $t$ - $(u$-) channel photon exchange, the amplitude is singular in the backward (forward) direction. This is a very common feature. It can be seen in fundamental equations as the classical Rutherford formula for Coulomb scattering.

The correct way to solve this problem, is by noting that the massless photon has an infinite range and that therefore the wavefunctions of the incoming vector bosons are not plane waves but Coulomb waves. But for our purpose, a simpler approach will be sufficient.

If in the CMS frame, $\theta$ is the angle between the incoming and the outgoing particle, we must integrate the amplitude from $\theta=0$ to $\theta=\pi$. In our calculation, the infinity can be regulated by introducing a small cut-off angle $\theta_{\text {cut }}$ and integrate the amplitude from $\theta_{\text {cut }}$ to $\pi$ (while taking the amplitude constant in the interval $\theta=0$ to $\theta=\theta_{\text {cut }}$ ). Alternatively, the divergence can be removed by giving the photon a small mass $\lambda_{\text {reg. }}$. These manipulations make the divergent term in the partial wave look like

$\log \left(\frac{1}{2} \theta_{\text {cut }}\right)^{2}$ respectively $\log \left(\frac{\lambda_{\text {Teg }}^{2}}{s-4 M^{2}+\lambda_{\text {reg }}^{2}}\right)$.

We will use the second regulator and set $\lambda_{\text {reg }}=1 \mathrm{keV}$. In the non-divergent terms of the partial wave, the photon mass is of course strictly zero.

Next, we must determine from which scattering amplitudes we will subtract limits on $\kappa$. We consider scatterings between neutral pairs of particles. Discarding all leptons, the only pairs are (with their proper normalization factors $): W_{L}^{+} W_{L}^{-},(1 / \sqrt{2}) W_{L}^{0} W_{L}^{0}, W_{L}^{0} H,(1 / \sqrt{2}) H H$.

We have verified that including the last two pairs does not tighten the limits on $\kappa$. The largest eigenvalue of the $4 \times 4$ partial wave matrix containing the channels $W_{L}^{+} W_{L}^{-}, W_{L}^{0} W_{L}^{0}, W_{L}^{0} H, H H$ is virtually the same as the largest eigenvalue of the $2 \times 2$ matrix containing only the $\mathrm{W}_{\mathrm{L}}^{+} \mathrm{W}_{\mathrm{L}}^{-}$and $\mathrm{W}_{\mathrm{L}}^{0} \mathrm{~W}_{\mathrm{L}}^{0}$ channels.

The partial waves of scatterings involving an external Higgs boson are small, because

(1) There are no terms, that grow with $s$. ( $\kappa$ does not appear in these amplitudes.)

(2) There are no propagators in these amplitudes that can become large for certain energies. Because of kinematical constraints, for no value of $\sqrt{s}$ can the pole of any propagator be reached.

So in this paper, we will only work with the $2 \times 2$ matrix of the $\mathrm{W}_{\mathrm{L}}^{+} \mathrm{W}_{\mathrm{L}}^{-}$and $\mathrm{W}_{\mathrm{L}}^{\mathrm{O}} \mathrm{W}_{\mathrm{L}}^{0}$ channels.

All the amplitudes and partial waves have been computed using the algebraic manipulation program SCHOONSCHIP. We now evaluate the partial waves in matrix (11) and use eq. (12) to set constraints on $\kappa$. 


\section{Results}

Fig. 4 depicts plots of $\kappa$ versus $\sqrt{s_{\text {weak }}}$ for various Higgs masses. In fig. 5 the critical energy $\sqrt{s_{\text {crit }}}$ is plotted as a function of the Higgs mass.

Fig. 4 shows, that the curves peak around $\kappa=1$, which is quite similar to fig. 3. Note that the curves are not defined around $\sqrt{s} \approx m$, because of the Higgs pole. That is why we left out the middle pieces of the curves in the case $m=400 \mathrm{GeV}$. The same effect is seen in fig. 5. For $m \ll \sqrt{s_{\text {crit }}}$ and $m \gg \sqrt{s_{\text {crit }}}$, the critical energy $\sqrt{s_{\text {crit }}}$ is almost constant. But if $m$ is close to $\sqrt{s_{\text {crit }}}$, our calculations do not give reliable results.

It is obvious what causes the problem. Our tree level expressions for the partial waves are not valid when $\sqrt{s} \approx m$, because there is a Higgs resonance at this energy. The missing part of a curve will be some interpolation between the two broken parts.

We can get an idea of how this would happen, by modifying the Higgs propagator like

$\frac{1}{-p^{2}+m^{2}} \rightarrow \frac{1}{-p^{2}+m^{2}-\mathrm{i} m \Gamma}$.

Not trying to be very accurate, we take the new parameter $\Gamma$, the width of the Higgs, as a simple constant.

We will show the effect of substitution (17) for fig. 5 . The dotted curve is found by taking $\Gamma=70 \mathrm{GeV}$. This is the smallest value for the width which keeps the resonance from saturating unitarity. We find that the critical energy changes very little (about $10 \%$ ) over the entire range of Higgs masses. The effect of taking a bigger width is that $\sqrt{s_{\text {crit }}}$ varies even less. So we conclude that if we discard the resonance, the critical energy is virtually constant at $\sqrt{s_{\text {crit }}} \approx 320 \mathrm{GeV}$.

At this place, it should be checked how different choices for the regulator $\lambda_{\text {reg }}$ affect the results. In fig. 6 the critical energy $\sqrt{s_{\text {crit }}}$ is plotted against $\log \left(\lambda_{\text {reg }}[\mathrm{eV}]\right)$. The Higgs mass is $m=500 \mathrm{GeV}$. (Taking a different mass only causes the curve to move up or down a little.) We find that $\sqrt{s_{\text {crit }}}$ changes only moderately as a function of $\lambda_{\text {reg. }}$. When $\lambda_{\text {reg }}$ changes 20 orders of magnitude, $\sqrt{s_{\text {crit }}}$ changes about $20 \%$.

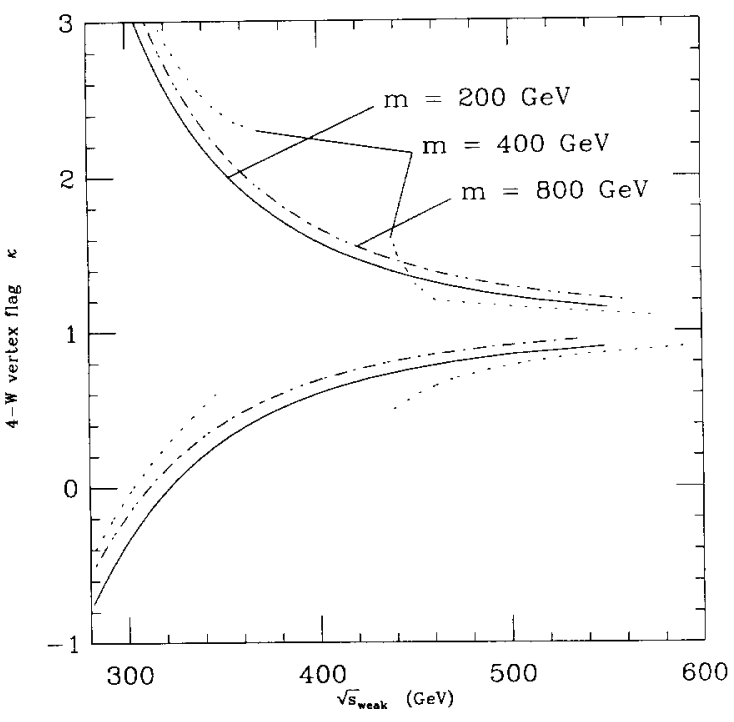

Fig. 4. Limits on $\kappa$ for different Higgs masses $m_{\text {Higgs }}=200,400$, $800 \mathrm{GeV}$.

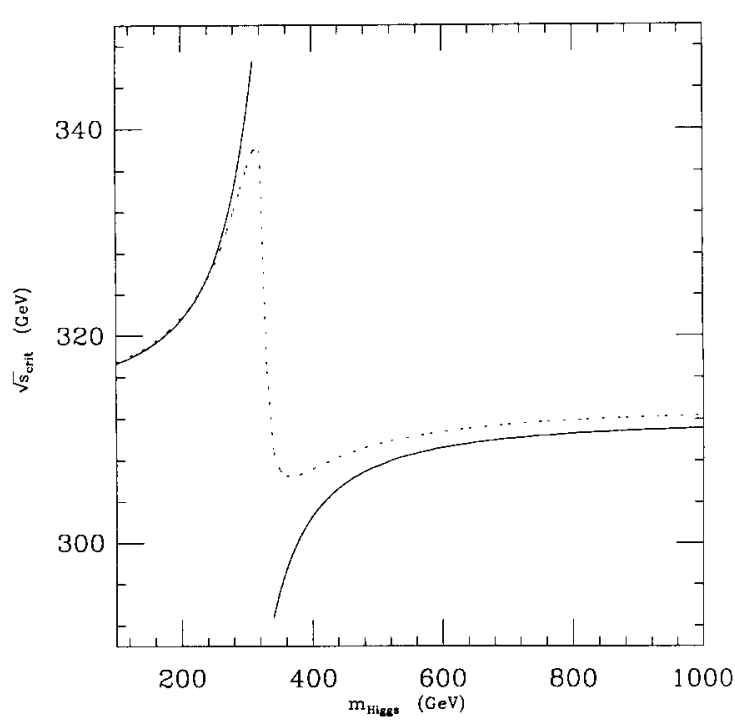

Fig. 5. The critical energy as a function of the Higgs mass. Solid curve: the Higgs has no width; broken curve: the Higgs has a width $\Gamma=70 \mathrm{GeV}$. 


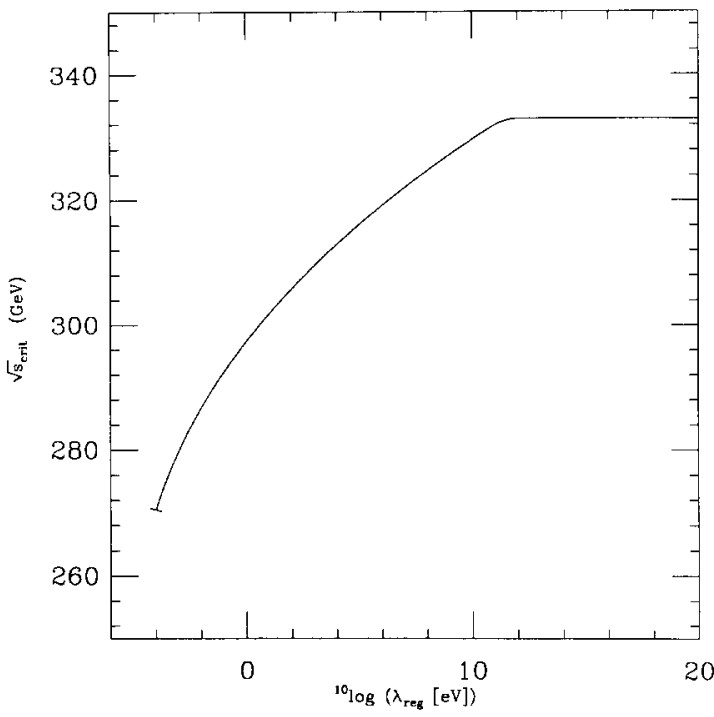

Fig. 6. The effect of $\lambda_{\text {reg }}$ on the critical energy $\left(m_{\text {Higgs }}=500 \mathrm{GeV}\right)$.

From fig. 6 , it must be decided which value for $\lambda_{\text {reg }}$ is reasonable. It should be picked so that the effect of the logarithm (16) is not extremely large or extremely small. To achieve this, we take $\lambda_{\text {reg }}$ to be somewhere halfway up on the slope. This corresponds to a value of $\lambda_{\text {reg }}$ around $1 \mathrm{keV}$.

\section{Conclusions}

We modified the vector boson 4-point vertices by adding a factor $\kappa$. The requirement that at tree level, unitarity is not violated up to an CMS energy $\sqrt{s_{\text {weak }}}$, puts limits on $\kappa$. The smallest energy for which this requirement implies that $\kappa>0$, is called the critical energy.

In the "high energy limit" (13), the critical energy is $\sqrt{s_{\text {crit }}}=320 \mathrm{GeV}(15)$. This is very compatible with the exact calculation. From fig. 5: $\sqrt{s_{\text {crit }}} \approx 300-350 \mathrm{GeV}$, slightly dependent on the Higgs mass. We also did the calculation using the less strict constraint $\left|a_{0}^{\mathrm{L}}(s)\right| \leqslant 1$. The results (graphs 4,5 ) were similar and we found $\sqrt{s_{\text {crit }}^{\prime}} \approx 400 \mathrm{GeV}$.

However, one has to be careful in the case of a very heavy Higgs. Even with $\kappa=1$, there comes a point where the tree level amplitude violates unitarity. When $m^{2} \gg s$, this happens at $\sqrt{s}=1.7 \mathrm{TeV}[1,4]$. It also turns out that including 1-loop radiative corrections to the WW scattering process, does not tighten these limits. In fact, the value of $\sqrt{s}$ where unitarity violation starts gets pushed down [5]. So it is not clear that adding 1-loop corrections in our calculations will result in tighter limits on $\kappa$.

Finally, we note that we did not give the $4 \mathrm{~W}$ vertex a completely general form like $\kappa\left(2 \delta_{\mu \nu} \delta_{\lambda \sigma}-\xi \delta_{\mu \lambda} \delta_{\nu \sigma}-\right.$ $\left.\xi^{\prime} \delta_{\mu \sigma} \delta_{\nu \lambda}\right)$. This would not be very useful, because with only one observable $\left(\sqrt{s_{\text {weak }}}\right)$, one cannot put restrictions on three parameters. Moreover, the critical energy does not depend on the structure of the $4 \mathrm{~W}$ vertex. Indeed, it is determined as the energy for which the partial waves of diagrams with only 3-point vertices saturate unitarity. From these considerations, it seems satisfactory to set $\xi=\xi^{\prime}=1$ and carry out the calculation in the presented manner. 


\section{Acknowledgement}

The author wants to thank M. Veltman for suggesting the problem and R. Akhoury and R. Bouamrane for many helpful discussions.

\section{References}

[1] B. Lee, C. Quigg and H. Thacker, Phys. Rev. D 16 (1977) 1519.

[2] G. Passarino and M. Veltman, Nucl. Phys. B 160 (1979) 151.

[3] M. Lüscher and P. Weisz, Phys. Lett. B 212 (1988) 472.

[4] M. Chanowitz and M. Gaillard, Nucl. Phys. B 261 (1985) 379.

[5] S. Dawson and S. Willenbrock, Phys. Rev. Lett. 62 (1989) 1232;

M. Veltman and F.J. Yndurain, Nucl. Phys. B 325 (1989) 1;

R. Bouamrane, preprint UM-TH-89-14. 